\title{
Angstzustände im Fremdsprachenunterricht von Studierenden der Abteilung Deutsch als Fremdsprache auf Lehramt (Am Beispiel Anadolu Universitåt)
}

\section{Students' Foreign-Language Classroom Anxiety at the Department of German Language Teaching Program (a Sample from Anadolu University)}

\author{
Şengül BALKAYA ${ }^{1}$ (D), Behiye ARABACIOĞLU2 ${ }^{(1)}$, Mustafa ÇAKIR $^{3}$ (1)
}

${ }^{1}$ Arș. Gör., Anadolu Üniversitesi, Eğitim Fakültesi, Yabancı Diller Eğitimi Bölümü, Eskişehir, Türkiye

${ }^{2}$ Dr. Öğr. Üyesi, Anadolu Üniversitesi, Eğitim Fakültesi, Yabancı Diller Eğitimi Bölümü,

Eskişehir, Türkiye

${ }^{3}$ Prof. Dr., T.C. Münih Başkonsolosluğu Eğitim Ataşesi, Münih, Âlmanya

ORCID: S..B. 0000-0002-0128-5103,

B.A. 0000-0002-5868-5319;

M.Ç. 0000-0002-0503-8564

\section{Corresponding author:}

Sengül BALKAYA

Anadolu Üniversitesi, Eğitim Fakültesi,

Yabancı Diller Eğitimi Bölümü, Eskişehir,

Türkiye

E-mail: sengulbalkaya@anadolu.edu.tr

Submitted: 21.08.2020

Revision Requested: 23.10 .2020

Last Revision Received: 28.10.2020

Accepted: 06.11.2020

Citation: Balkaya, S., Arabacioglu, B., \&

Cakir, M. (2020). Angstzustände im

Fremdsprachenunterricht von

Studierenden der Abteilung Deutsch als

Fremdsprache auf Lehramt (Am Beispiel

Anadolu Universität). Alman Dili ve

Edebiyatı Dergisi - Studien zur deutschen

Sprache und Literatur, 44, 111-134.

https://doi.org/10.26650/sds|2020-0019

\begin{abstract}
DEUTSCH)
Die vorliegende Studie wurde durchgeführt, um die Angstzustände im Fremdsprachenunterricht von Studierenden der Abteilung Deutsch als Fremdsprache auf Lehramt zu ermitteln und die genannten Zustände im Fremdsprachenunterricht anhand verschiedener Variablen zu untersuchen. In der Untersuchung wurde das Survey-Modell verwendet. Insgesamt 70 Studierende, die an der Anadolu Universität Deutsch als Fremdsprache studieren, bilden die Stichprobe der Untersuchung. Die aus der Studie erhaltenen quantitativen Daten wurden mit der "Befragung zur Angst im Fremdsprachenunterricht" von Horwitz gesammelt, die er mit Horwitz und Cope zusammen (1986) entwickelt hat und die von Aydın und Zengin (2008) ins Türkische übertragen wurde. Die qualitativen Daten dagegen wurden mit einem halbstrukturierten Interviewformular gesammelt. Zur Analyse der Daten wurden deskriptive Statistiken, eine EinwegVarianzanalyse (One-Way-ANOVA) und eine Inhaltsanalyse verwendet. Aus den Ergebnissen der Studie geht hervor, dass die Fremdsprachenangst der Studierenden auf einem mittleren Niveau liegt. Darüber hinaus wurde der Entschluss gezogen, dass sich das Geschlecht der Studierenden, die Art des besuchten Gymnasiums und das Sprachniveau nicht auf die Angstzustände auswirkt, wohingegen ein Aufenthalt im Ausland, ein Auslandssemester und ein Vorbereitungsjahr an einer Universität erhebliche Auswirkungen auf die Angstzustände aufzeigt. Außerdem wurde festgestellt, dass die Gründe für die Angst der Studierenden durch externe Faktoren wie Gruppenzwang sowie interne Faktoren wie Angst vor Fehlverhalten, mangelndem Selbstvertrauen und Scham in Bezug auf Grammatik, Wortauswahl und Aussprache verursacht wurden.
\end{abstract}

Schlüsselwörter: Angst, Angstzustände im Fremdsprachenunterricht, Fremdsprache, Deutsch als Fremdsprache, Fremdsprache auf Lehramt 


\begin{abstract}
ENGLISH)
This study aimed to assess students' anxiety levels at the Department of German Language Teaching Program at Anadolu University and examine foreign-language teaching conditions based on various variables. It adopted a survey model and recruited a sample of 70 students from the said department. Quantitative data were obtained using the Questionnaire of Foreign Language Classroom Anxiety developed by Horwitz, Horwitz and Cope (1986) and adapted to Turkish by Aydın and Zengin (2008). Qualitative data, on the other hand, were collected from semistructured interviews. For data analysis, this study applied descriptive statistics, one-way analysis of variance (ANOVA), and content analysis. The results showed that students have a medium level of anxiety in foreign languages, and while they receive foreign language lessons, they do not consider themselves competent enough. In addition, students' gender, type of high school attended, and language level did not influence their anxiety whereas their stay and study abroad and their preparatory year at a university significantly affected it. External factors, such as peer pressure, and internal factors, such as fear of wrongdoing, lack of self-confidence, and embarrassment regarding grammar, word choice, and pronunciation, were also found to contribute to student anxiety. Keywords: Anxiety, foreign language classroom anxiety, foreign language, German as a foreign language, foreign language teaching program
\end{abstract}

\title{
EXTENDED ABSTRACT
}

Modern times bring about many innovations that make life easier for society; along with these, other factors emerge that negatively affect life. It has also been established that people's intense work pace, economic conditions, and goals trigger a host of negative emotions such as stress, fear, anxiety, and concern. Research has also shown that these problems, which arise at life's every moment, adversely affect individuals by impeding their work and even their success. While this study views "Emotions in foreignlanguage lessons" as an overall topic, it specifically examines anxiety in the context of foreign-language lessons. The anxiety we encounter in different areas of life has now become an important issue in foreign-language courses, which necessitates an investigation of the negative factors that shape an individual from primary to higher education and, in this regard, the necessary measures for future foreign-language courses. Hence, this study was conducted to evaluate the anxiety levels of students at the Department of German Language Teaching Program and examine foreign-language teaching conditions using different variables. For this purpose, the following questions were asked:

1. What is the anxiety level regarding foreign-language teaching among students in the Department of German Language Teaching?

2. How do the students' anxiety levels change depending on
a. gender?
b. type of high school attended?
c. their stay abroad? 

d. their study abroad?
e. their attendance at the preparatory class at university?
$\mathrm{f}$. their proficiency in German as a foreign language in high school?

3. What factors influence students' anxious states regarding foreign-language teaching in the Department of German Language Teaching?

4. What activities can be implemented to mitigate students' anxiety in the Department of German Language Teaching Program?

To establish the study's validity and reliability, a survey model was carried out. A total of 70 students studying at the Department of German Language Teaching Program at Anadolu University were recruited for the study. Quantitative data were gathered using the Questionnaire of Foreign Language Classroom Anxiety, which was developed by Horwitz, Horwitz and Cope (1986) and adapted into Turkish by Aydın and Zengin (2008). Meanwhile, qualitative data were collected from semistructured interview sessions, which were organized by the authors and included the following questions:

1. Do you feel anxiety while speaking in a foreign-language classroom? Please explain.

2. What are the reasons for the negative effect of anxiety on your language learning?

3. Are there any measures taken in your foreign-language courses that mitigate your language anxiety? If yes, what are they?

4. What are the factors that positively affect your foreign-language learning?

The data analysis applied descriptive statistics and one-way analysis of variance (ANOVA) for the quantitative data and content analysis for the qualitative data. The results showed that students' anxiety in foreign languages is at a medium level, and although they receive foreign language lessons, they do not consider themselves sufficiently competent. Also, the participants' gender, type of high school attended, and language level did not affect their anxiety; meanwhile, their stay and study abroad and their preparatory year at a university had significant effects on their anxious states. In addition, student anxiety was caused by external factors, such as peer pressure, and internal factors, such as fear of wrongdoing, lack of self-confidence, and embarrassment regarding grammar, word choice, and pronunciation. After a general assessment of the research results, it became clear that education faculties need to be redesigned and improved so that the anxiety states of intermediate-level students in the Department of German Language Teaching Program are reduced to a minimum. 


\section{Einleitung}

Es ist bekannt, dass die moderne Zeit viele Innovationen mit sich bringt, die das Leben der Gesellschaften erleichtern, jedoch zugleich auch einige Faktoren mit sich bringt, die sich negativ auf das Leben auswirken. Auch ist es eine bekannte Tatsache, dass ein intensives Arbeitstempo, wirtschaftliche Bedingungen und Ziele der Menschen einige negative Verhaltensweisen wie Stress, Angst und Besorgnis mit sich bringen. Diese Probleme, die in jedem Moment des Lebens auftreten, wirken sich laut Untersuchungen negativ auf den Einzelnen aus, indem seine Arbeit und sogar sein Erfolg verhindert werden. Als Gesamtthema könnte hier „Emotionen im Fremdsprachenunterricht" betrachtet werden, jedoch wird in der vorliegenden Studie die Angst im Rahmen des Fremdsprachenunterrichts unter die Lupe genommen. Die Angst, der man in verschiedenen Lebensbereichen begegnet, ist heute auch im Fremdsprachenunterricht zu einem wichtigen Problem geworden. Es ist von großer Bedeutung, den Faktor, der eine Person von seiner Grundschulbildung bis zur Hochschulbildung negativ beeinflusst, zu untersuchen und in dieser Hinsicht die notwendigen Maßnahmen für den zukünftigen Fremdsprachenunterricht zu treffen.

Während im Allgemeinen Angst als „Traurigkeit, besorgter Gedanke, Sorge“ definiert wird (TDK, Institution für türkische Sprache), beschreiben Aydın und Zengin (2008, S. 84) die Angst als „einen emotionalen Zustand mit wahrgenommener Schwäche während der Vorbereitung auf eine wahrgenommene Gefahr“. Der negativ angesehene Begriff Angst zeigt sich im Lernprozess jedoch mit anderen Auswirkungen. In der Studie über die Auswirkungen von Angstzuständen im Fremdsprachenunterricht, kommt Genç (2009) zu der Erkenntnis, dass sehr niedrige oder sehr hohe Angstzustände das Lernen negativ beeinflussen und moderate Angstzustände das Lernen sogar positiv beeinflussen. In den letzten Jahren ist die Angst, die im allgemeinen Studienbereich der Psychologie anzusiedeln ist, auch in anderen Bereichen zum Forschungsgegenstand geworden, dazu gehören insbesondere die Forschungen im Fremdsprachenunterricht. Laut Horwitz, Horwitz und Cope (1986) wirkt sich die Angst, die in den letzten Jahren zu den größten Problemen im Fremdsprachenunterricht geworden ist, negativ auf das Lernen aus. Zahlreiche Studien sind durchgeführt worden, um die Gründe der Angst vor dem Sprechen einer Fremdsprache aufzudecken. Während Bekleyen (2004) die Wirkung von Lehrern und Klassenkameraden auf die Angstbildung untersuchte, untersuchten Batumlu und Erden (2007), Şener (2015), Doğan und Tuncer (2016) die Auswirkung von Angst auf den Erfolg und kamen zu dem Entschluss, dass Angst einen negativen Einfluss 
auf den Erfolg hat. Balemir (2009) untersuchte die Ursachen von Sprachangst und den Zusammenhang zwischen Angstniveau und Kompetenzen. Auch Gedik (2015) und Öner und Gedikoğlu (2007) untersuchten die Ängste von Schülern der Sekundarstufe in Bezug auf das Sprechen von Englisch als Fremdsprache, dagegen bezog sich Önem (2010) in seiner Studie auf die Angst von Zuständen und Kontinuität. Öztürk und Gürbüz (2014) sowie Tüm und Kunt (2013) bestimmten die Sprachangst der Schüler im Englischunterricht. Zerey (2008) hat als Resultat seiner Masterarbeit vorgelegt, dass Sprachangst durch Theaterpraxis reduziert werden kann. In ähnlicher Weise begründet Zhanibek (2001), dass es einen signifikanten Zusammenhang zwischen Unterrichtsbeteiligung und Sprachangst gibt. Es ist deutlich, dass viele Studien über die Angstzustände im Fremdsprachenunterricht auf den Englischunterricht und auf die Sekundarstufe basieren. Darüber hinaus betonten İşigüzel und Kırmızı (2014) in ihrer Studie, dass die Angst ein wichtiger Faktor im Deutschunterricht der Sekundarstufe ist, der den Erfolg der Schüler im Fremdsprachenunterricht beeinflusst. In ähnlicher Weise kam Can (2014) zu dem Entschluss, dass die Angst vor dem Sprechen das Hauptproblem beim Erlernen einer zweiten Fremdsprache ist. Als erste Studie im Bereich Deutsch als zweite Fremdsprache auf Lehramt, sind Yilmaz und Maden (2016) zu betrachten, die eine Untersuchung über das allgemeine Angstniveau von Studierenden der Abteilung Deutsch als Fremdsprache auf Lehramt durchgeführt haben. Sie haben den Entschluss gezogen, dass die Angstzustände der Studierenden höher sind und sich somit in Bezug auf einen Aufenthalt im Ausland ändern. Als letztes untersuchte Oflaz (2019) in seiner Arbeit die Beziehung zwischen Angst, dem Einsatz von Sprachlernstrategien und den akademischen Leistungen von Studierenden der Vorbereitungsklasse Deutsch als Fremdsprache. Dabei kam er zu dem Entschluss, dass Studierende, die weniger Angst haben, im akademischen Bereich erfolgreicher sind als die, deren Angstniveau höher anliegt.

Es gibt Studien in der internationalen Literatur zur Angst vor dem Fremdsprachenunterricht (Aida, 1994; Azher, Anwar \& Naz, 2010; Dörnyei, 1994; Fischer 2005; Kim, 2009; Matsuda \& Gobel, 2004; Rodríguez \& Abreu, 2003; Sparks \& Ganschow, 2007; Trylong, 1987; Young, 1991). Sowie auch in allgemeinen türkischen wissenschaftlichen Bereichen sind verschiedene Studien in Zusammenhang mit Angst vor dem Fremdsprachenunterricht verfügbar (Balemir, 2009; Batumlu \& Erden, 2007; Bekleyen, 2004; Can, 2014; Doğan \& Tuncer, 2016; Duman, Göral \& Bilgin, 2017; Gedik, 2015; İşigüzel \& Kırmızı, 2014; Oflaz, 2019; Önem, 2010; Öner \& Gedikoğlu, 2007; Öztürk \& Gürbüz, 2014; Şener, 2015; Tüm \& Kunt, 2013; Zerey, 2008). 
Es wurde festgestellt, dass im Bereich Deutsch als Fremdsprache auf Lehramt Angstzustand nicht genügend erforscht worden ist. Daher ist das Ziel der vorliegenden Studie die Faktoren, die die Angst und das Angstniveau der Studierenden der Abteilung Deutsch als Fremdsprache auf Lehramt der Anadolu Universität im Bereich des Deutschunterrichts beeinflussen, zu bestimmen und darüber hinaus am Ende der Studie den Ergebnissen entsprechend einige Vorschläge zu machen.

Zu diesem allgemeinen Zweck wurden folgende Fragen gestellt:

1. Wo liegt das Angstniveau im Fremdsprachenunterricht bei Studierenden der Abteilung für Deutsch als Fremdsprache auf Lehramt?

2. Ändert sich das Angstniveau bei den Studierenden je nach

a. Geschlecht

b. Art des besuchten Gymnasiums

c. Aufenthalt im Ausland

d. Auslandssemester

e.Besuch der Vorbereitungsklasse an der Universität

f. Sprachniveau von Deutsch als Fremdsprache im Gymnasium

3. Welche Faktoren beeinflussen die Angstzustände im Fremdsprachenunterricht von Studierenden der Abteilung für Deutsch als Fremdsprache auf Lehramt?

4. Gibt es Maßnahmen darüber, wie die Angst der Studierenden der Abteilung für Deutsch als Fremdsprache auf Lehramt im Fremdsprachenunterricht beseitigt werden kann?

\section{Methode}

In diesem Teil der Studie werden das angewandte Forschungsdesign und die Arbeitsgruppe, die Datenerhebungstechniken und die Datenanalyse dargelegt.

\subsection{Das Forschungsdesign}

Diese Studie wurde entwickelt, um die Ängste der Studierenden der Abteilung Deutsch als Fremdsprache auf Lehramt an der Pädagogischen Fakultät der Anadolu Universität zu untersuchen. Sie basiert auf dem Survey Modell und um sich gegenseitig zu stützen werden, sowohl quantitative als auch qualitative Daten verwendet. Die aus der Studie erhaltenen quantitativen Daten wurden mit der „Befragung der Angst vor dem 
Fremdsprachenunterricht" von Horwitz gesammelt, die er mit Horwitz und Cope zusammen (1986) entwickelt hat und die von Aydın und Zengin (2008) ins Türkische übertragen wurde. Außerdem wurden die qualitativen Daten durch semistrukturierte Interviews gesammelt. Um eine gegenseitige Stützung zu gewährleisten und mehr Informationen zu erhalten, ist es üblich, quantitative und qualitative Daten häufig zusammen zu erheben (Dörnyei 2007, zit. nach Settinieri und andere, 2014, S. 21).

\subsection{Teilnehmer der Studie}

Die Grundgesamtheit der Untersuchung besteht aus den Studierenden der Abteilung für Deutsch als Fremdsprache auf Lehramt der Pädagogischen Fakultät der Anadolu Universität. Die Teilnehmer der Studie sind 70 Studierende des zweiten Studienjahres, die nach einer überproportionalen Stichprobenmethode ausgewählt wurden. An der Studie haben ausschließlich freiwillige Studierende teilgenommen. Im Folgenden zeigt Tabelle 1 die demographischen Merkmale der Studierenden dieser Untersuchung.

Tabelle 1: Aufteilung der Teilnehmer nach demografischen Variablen

\begin{tabular}{|l|l|c|c|}
\hline Variablen & Kategorien & N & \% \\
\hline \multirow{5}{*}{ Geschlecht } & 1. weiblich & 46 & 65.7 \\
\cline { 2 - 4 } & 2. männlich & 24 & 34.3 \\
\hline \multirow{4}{*}{ Gymnasium } & 1. Naturwissenschaftliches Gymnasium & 0 & 0 \\
\cline { 2 - 4 } & 2. Anadolu Gymnasium & 56 & 80.0 \\
\cline { 2 - 4 } & 3. Fachgymnasium & 2 & 2.9 \\
\cline { 2 - 4 } & 4. Andere & 12 & 17.1 \\
\hline \multirow{2}{*}{ Aufenthalt im Ausland } & Ja & 22 & 31.4 \\
\hline \multirow{2}{*}{ Auslandssemester } & Nein & 48 & 68.6 \\
\hline \multirow{2}{*}{ Vorbereitungsklasse an der Uni } & Ja & 12 & 17.1 \\
\hline \multirow{2}{*}{$\begin{array}{l}\text { Deutschkenntnisniveau } \\
\text { im Gymnasium }\end{array}$} & Nein & 58 & 82.9 \\
\hline & Nein & 8 & 88.6 \\
\hline & schlecht & 31 & 11.4 \\
\hline & befriedigend & 30 & 44.3 \\
& gut & 9 & 42.9 \\
\hline
\end{tabular}

Bei der Betrachtung von Tabelle 1 zeigt sich, dass 65,7\% der Teilnehmer weiblich ( $\mathrm{N}=$ 46) und 34,3\% männlich ( $N=24)$ sind. Darüber hinaus geben $0 \%$ der Teilnehmer an, dass sie das naturwissenschaftliche Gymnasium abgeschlossen haben ( $N=0)$ (daher nicht in anderen Analysen enthalten). 80,0\% ( $N=56$ ) geben an, das anatolische Gymnasium abgeschlossen zu haben, 2,9\% $(\mathrm{N}=2)$ das Fachgymnasium und die restlichen Teilnehmer 
$17,1 \%(\mathrm{~N}=12)$ andere Gymnasien absolviert haben. Darüber hinaus geben 31,4\% der Studierenden an, im Ausland gewesen zu sein $(N=22)$ (diese Studierende sind im Ausland geboren und/oder aufgewachsen), während 68,6\% angegeben haben, nicht im Ausland gewesen zu sein ( $\mathrm{N}=48) .17,1 \%$ der Studierenden haben Auslandssemester absolviert ( $\mathrm{N}=12)$, während $82,9 \%(\mathrm{~N}=58)$ keine Auslandssemester absolviert haben. Ein weiteres Ergebnis der Studie ist, dass $88,6 \%$ der Studierenden eine Vorbereitungsklasse besucht haben $(\mathrm{N}=62)$ und $11,4 \%(\mathrm{~N}=8)$ keine Vorbereitungsklasse besucht haben. Außerdem wurde den Studierenden die Frage gestellt, wie sie ihr eigenes Deutschkenntnisniveau im Gymnasium bewerten. Die letzte demografische Variable, die sich aus der Studie ergibt, ist, dass $44,3 \%$ der Studierenden angeben, dass ihr Deutschniveau am Gymnasium ( $N=31$ ) schlecht sei, während 42,9\% der Studierende angeben, dass ihr Deutschniveau am Gymnasium $(\mathrm{N}=30)$ befriedigend sei und $12,8 \%$. ( $N$ $=9)$, dass es gut sei.

\subsection{Datenerhebungsmittel}

Die Studierenden wurden mit einer Befragung befragt, deren 5-stufige Antwortskala von „trifft voll und ganz zu“ (1) bis „trifft überhaupt nicht zu“ (5) reicht. Somit beträgt die höchste Durchschnittspunktzahl 5 und die niedrigste Durchschnittspunktzahl 1 für jedes Item. Die eindimensionale Befragung besteht aus 33 Items. 24 Items in dieser Befragung zielen auf die Angst der Studierenden gegenüber der Fremdsprache, während 9 Items die Angst mit negativen Ausdrücken messen. Der aus der Studie erhaltene AlphaZuverlässigkeitskoeffizient von Cronbach wurde mit 0,94 berechnet. Qualitative Daten wurden über ein semistrukturiertes Interviewformular mit 4 Fragen gesammelt, die von den Forschern entwickelt wurden, um detailliertere Informationen zu erhalten.

\subsection{Datenanalyse}

Bei der Auswertung der quantitativen Daten aus der Studie wurde das Statistical Package for Social Sciences (SPSS für Windows Version 20.0) verwendet. Zuerst wurden deskriptive Statistiken verwendet, um die quantitativen Daten zu analysieren. Um zu testen, ob sich die Ängste der Studierenden der Abteilung Deutsch als Fremdsprache auf Lehramt den Variablen entsprechend geändert haben, wurde der t-Test in binären Variablen verwendet. Für mehr als zwei Variablen wurden Vergleiche mit One-WayANOVA durchgeführt. Als Signifikanzniveau wurde in allen Berechnungen 0,05 angenommen. Bei der Analyse der qualitativen Daten wurde die Inhaltsanalyse, die als 
deskriptive Analysetechnik gilt, verwendet. Die Meinungen der Studierenden zu den Fragen wurden direkt mit numerischen Codes wie S1, S2, S3 (Studierender 1, Studierender $2, \ldots)$ dargestellt.

\section{Befunde}

\subsection{Befunde der Befragung}

Im folgenden Teil der Studie werden die Angstzustände der Studierenden der Abteilung Deutsch als Fremdsprache auf Lehramt dargestellt und es wird untersucht, ob sich die Angstzustände in Bezug auf verschiedene Variablen ändern oder nicht, auch die aus qualitativen Fragen gewonnenen Ergebnisse werden in diesem Teil präsentiert. Die erste Forschungsfrage der Untersuchung beschäftigt sich mit der Frage, ob die Studierenden der Abteilung Deutsch als Fremdsprache auf Lehramt der Anadolu Universität Angst vor dem Sprechen im Fremdsprachenunterricht haben. Zu diesem Zweck wurde die Durchschnittsbewertung der Befragung ermittelt und in der folgenden Tabelle 2 dargestellt.

Tabelle 2: Mittelwert der Angstwerte von Studierenden

\begin{tabular}{|l|c|c|c|c|c|}
\hline Angstwerte im Fremdsprachenunterricht & N & Min. & Max. & $\overline{\mathbf{X}}$ & $\mathbf{S}$ \\
\hline Gesamt & 70 & 1.70 & 4.67 & 3.17 & 0.77 \\
\hline
\end{tabular}

Betrachtet man Tabelle 2, so zeigt sich, dass die Fremdsprachenangst der Studierenden auf einem mittleren Niveau liegt ( $\bar{X}=3.17, S=0.77)$. Mit anderen Worten, die Ängste der Studierenden im Fremdsprachenunterricht sind weder sehr hoch noch sehr niedrig.

Um die Frage „Welche Angstzustände im Fremdsprachenunterricht bestehen bei Studierenden der Abteilung Deutsch als Fremdsprache auf Lehramt?" zu beantworten, werden beschreibende Analysen (arithmetische Mittel und Standardabweichung) der einzelnen Elemente der Befragung in Tabelle 3 aufgeführt.

Tabelle 3: Deskriptive Analyse der Angstwerte von Studierenden der Abteilung Deutsch als Fremdsprache auf Lehramt

\begin{tabular}{|l|c|c|c|c|c|}
\hline Items & $\mathbf{N}$ & Min. & Max. & $\overline{\mathbf{X}}$ & S \\
\hline I 1 & 70 & 1 & 5 & 2.82 & 1.21 \\
\hline I 2 & 70 & 1 & 5 & 2.72 & 1.25 \\
\hline I 3 & 70 & 1 & 5 & 3.00 & 1.34 \\
\hline
\end{tabular}




\begin{tabular}{|c|c|c|c|c|c|}
\hline 14 & 70 & 1 & 5 & 2.87 & 1.32 \\
\hline 15 & 70 & 1 & 5 & 3.78 & 1.41 \\
\hline 16 & 70 & 1 & 5 & 2.73 & 1.22 \\
\hline 17 & 70 & 1 & 4 & 3.14 & 1.38 \\
\hline 18 & 70 & 1 & 5 & 3.24 & 1.18 \\
\hline 19 & 70 & 2 & 5 & 2.61 & 1.33 \\
\hline 110 & 70 & 1 & 5 & 2.50 & 1.22 \\
\hline I 111 & 70 & 1 & 5 & 2.91 & 1.29 \\
\hline 112 & 70 & 1 & 5 & 3.24 & 1.22 \\
\hline 113 & 70 & 1 & 5 & 3.33 & 1.32 \\
\hline 114 & 70 & 1 & 5 & 3.51 & 1.39 \\
\hline 115 & 70 & 1 & 5 & 3.15 & 1.31 \\
\hline 116 & 70 & 1 & 5 & 3.38 & 1.19 \\
\hline 117 & 70 & 1 & 5 & 3.30 & 1.40 \\
\hline 118 & 70 & 1 & 5 & 2.97 & 1.23 \\
\hline 119 & 70 & 1 & 5 & 3.54 & 1.13 \\
\hline 120 & 70 & 1 & 5 & 3.00 & 1.25 \\
\hline 121 & 70 & 1 & 5 & 3.60 & 1.22 \\
\hline 122 & 70 & 1 & 5 & 3.02 & 1.22 \\
\hline 123 & 70 & 1 & 5 & 3.05 & 1.40 \\
\hline 124 & 70 & 1 & 5 & 3.05 & 1.38 \\
\hline 125 & 70 & 1 & 5 & 3.61 & 1.14 \\
\hline 126 & 70 & 1 & 5 & 3.67 & 1.23 \\
\hline 127 & 70 & 1 & 5 & 3.24 & 1.13 \\
\hline 128 & 70 & 1 & 5 & 3.17 & 1.20 \\
\hline 129 & 70 & 2 & 5 & 3.08 & 1.13 \\
\hline 130 & 70 & 1 & 5 & 3.15 & 1.19 \\
\hline 131 & 70 & 1 & 5 & 3.72 & 1.26 \\
\hline 132 & 70 & 1 & 5 & 2.84 & 1.41 \\
\hline 133 & 70 & 1 & 5 & 2.71 & 1.14 \\
\hline
\end{tabular}

Bei Betrachtung von Tabelle 3 wird ersichtlich, dass das Item, das den höchsten Wert aufweist, das Item 5 war $(\overline{\mathrm{X}}=3.78, \mathrm{~S}=1.41)$ "Es stört mich nicht, die Anzahl der Deutschstunden zu erhöhen“. Dem folgen Item $26(\bar{X}=3.67, S=1.23)$ "Ich fühle mich im Deutschunterricht angespannter als ich eigentlich bin" und Item $25(\bar{X}=3.61, S=1.14)$ „Der Deutschunterricht ist so schnell, dass ich befürchte, zurückzubleiben“. Auf der anderen Seite ist das Item mit dem niedrigsten Wert das Item $10(\bar{X}=2.50, S=1.22)$ mit dem Ausdruck „Ich mache mir Sorgen über Probleme, die auftreten können, wenn ich im Deutschunterricht keinen Erfolg habe". Diesem Item folgen Item $9(\bar{X}=2.61, S=1.33$ ) „Wenn ich im Deutschunterricht unvorbereitet sprechen muss, werde ich unruhig" und Item 33 ( $\overline{\mathrm{X}}=2.71, \mathrm{~S}=1.14)$ "Ich fühle mich unwohl, wenn der Deutschlehrer Fragen zu Themen stellt, auf die ich nicht vorbereitet bin." 
Eine Subfrage der Studie ist, ob sich die Angstwerte der Studierenden der Abteilung Deutsch als Fremdsprache auf Lehramt im Fremdsprachenunterricht je nach Geschlecht unterscheiden. Zur Beantwortung dieser Frage wurde ein t-Test für unabhängige Gruppen durchgeführt. Die Ergebnisse sind in Tabelle 4 aufgeführt.

Tabelle 4: Vergleich der Angstwerte der Studierenden je nach Geschlecht

\begin{tabular}{|l|c|c|c|c|c|c|}
\hline Variable & $\mathbf{n}$ & $\overline{\mathbf{X}}$ & $\mathbf{S}$ & $\mathbf{s d}$ & $\mathbf{t}$ & $\mathbf{p}$ \\
\hline weiblich & 46 & 3.02 & 0.80 & 68 & 1.78 & 0.08 \\
\hline männlich & 24 & 3.37 & 0.68 & & & \\
\hline
\end{tabular}

Gemäß den Ergebnissen aus Tabelle 4 wurde der Entschluss gezogen, dass sich die Angstwerte der Studierenden der Abteilung Deutsch als Fremdsprache auf Lehramt $\left(\mathrm{t}_{(68)}=1.78: \mathrm{p}<.05\right)$ je nach "Geschlecht" nicht ändern. Mit anderen Worten gab es keinen signifikanten Unterschied zwischen den Angstwerten weiblicher und männlicher Studierenden. Eine andere Variable, die in der Untersuchung getestet wurde, ist die Frage, ob sich die Angstwerte der Studierenden im Fremdsprachenunterricht in Bezug auf den Schultyp unterscheiden. Um diese Frage zu beantworten wurde One-Way-ANOVA durchgeführt, die Ergebnisse sind in Tabelle 5 dargestellt.

Tabelle 5: Vergleich der Angstwerte der Studierenden je nach Schultyp

\begin{tabular}{|l|c|c|c|c|c|c|}
\hline Variablen & $\mathbf{n}$ & $\overline{\mathbf{X}}$ & $\mathbf{S}$ & $\mathbf{s d}$ & $\mathbf{F}$ & $\mathbf{p}$ \\
\hline Gynasium & 56 & 3.09 & 0.74 & $2-67$ & 1.67 & 0.19 \\
\hline Fachgymnasium & 2 & 4.09 & 0.51 & & & \\
\hline Andere & 12 & 3.21 & 0.89 & & & \\
\hline
\end{tabular}

Wenn man Tabelle 5 näher betrachtet, so zeigt sich, dass die Fremdsprachenangstwerte der Studierenden sich nicht je nach dem Schultyp $[F(2-67)=1.67: p<0.05]$ unterscheiden. Mit anderen Worten sind die Angstwerte der Studierenden nicht vom Schultyp beeinflusst.

Darüber hinaus wird die Frage untersucht, ob die Angstwerte der Studierenden je nach Auslandsaufenthalt unterschiedlich sind. Die t-Testergebnisse zu dieser Frage sind in Tabelle 6 dargestellt.

Tabelle 6: Vergleich der Angstwerte der Studierenden je nach Auslandsaufenthalt

\begin{tabular}{|l|c|c|c|c|c|c|}
\hline Variable & $\mathbf{n}$ & $\overline{\mathbf{X}}$ & $\mathbf{S}$ & $\mathbf{s d}$ & $\mathbf{t}$ & $\mathbf{p}$ \\
\hline Ja & 22 & 3.61 & 0.72 & 68 & 3.72 & 0.00 \\
\hline Nein & 48 & 2.93 & 0.70 & & & \\
\hline
\end{tabular}


Bei der Untersuchung von Tabelle 6 wurde festgestellt, dass sich die Angstwerte der Studierenden im Fremdsprachenunterricht in Abhängigkeit von der Variable Auslandsaufenthalt $\left(\mathrm{t}_{(68)}=3.72: \mathrm{p}<.05\right)$ signifikant unterscheiden. Aus Tabelle 6 geht hervor, dass dieser signifikante Unterschied zugunsten der Studierenden zu sehen ist, die nicht im Ausland waren. Mit anderen Worten zeigt sich, dass die Angstwerte der Studierenden, die einen Aufenthalt im Ausland erlebt haben, höher sind als die derjenigen, die keinen solchen erlebt haben.

Zusammen mit dem Auslandsaufenthalt wurde auch untersucht, ob sich die Fremdsprachenangstwerte der Studierenden geändert haben, je nachdem ob sie ein Auslandssemester absolviert haben. Die t-Testergebnisse für diese Frage sind in Tabelle 7 aufgeführt.

Tabelle 7: Vergleich der Angstwerte von Studierenden je nach Auslandssemester

\begin{tabular}{|l|c|c|c|c|c|c|}
\hline Variablen & $\mathbf{n}$ & $\overline{\mathbf{X}}$ & $\mathbf{S}$ & $\mathbf{s d}$ & $\mathbf{t}$ & $\mathbf{p}$ \\
\hline Ja & 12 & 3.80 & 0.80 & 68 & 3.50 & 0.01 \\
\hline Nein & 58 & 3.00 & 0.70 & & & \\
\hline
\end{tabular}

An der Tabelle 7 ist deutlich zu sehen, dass die Angstwerte der Studierenden der Abteilung Deutsch als Fremdsprache auf Lehramt in Bezug auf ein Auslandssemester erheblich voneinander abweichen $\left(\mathrm{t}_{(68)}=3.50: \mathrm{p}<0.5\right)$. Aus Tabelle 7 geht hervor, dass dieser signifikante Unterschied für diejenigen spricht, die im Ausland zur Schule gegangen sind. Mit anderen Worten wird deutlich, dass die Fremdsprachenangstwerte der Studierenden, die im Ausland zur Schule gegangen sind, höher sind als die der Studierenden, die kein Auslandssemester absolviert haben.

In der Untersuchung wird auch die Variable der Studienvorbereitung an der Universität untersucht. Tabelle 8 zeigt die Ergebnisse des t-Tests, bei dem festzustellen war, ob sich die Angstwerte der Studierenden im Fremdsprachenunterricht in Bezug auf den Besuch einer Vorbereitungsklasse an der Universität variieren.

Tabelle 8: Vergleich der Angstwerte von Studierenden je nach Besuch einer Vorbereitungsklasse an der Universität

\begin{tabular}{|l|c|c|c|c|c|c|}
\hline Variable & $\mathbf{n}$ & $\overline{\mathbf{X}}$ & $\mathbf{S}$ & $\mathbf{s d}$ & $\mathbf{t}$ & $\mathbf{p}$ \\
\hline Ja & 62 & 3.01 & 0.72 & 68 & 4.23 & 0.00 \\
\hline Nein & 8 & 4.12 & 0.41 & & & \\
\hline
\end{tabular}


In Tabelle 8 wird abgebildet, ob die Angstwerte im Fremdsprachenunterricht der Studierenden von einem Besuch der Vorbereitungsklasse abhängig sind oder nicht $\left(t_{(68)}=4.23: p<0.5\right)$. Aus Tabelle 8 geht hervor, dass dieser signifikante Unterschied für diejenigen spricht, die keine Vorbereitungsklasse besucht haben. Mit anderen Worten zeigt sich, dass die Angstwerte der Studierenden, die keine vorbereitende Ausbildung an der Universität absolviert haben, höher sind als die der Studierenden, die eine vorbereitende Ausbildung an der Universität erhalten haben.

Die letzte Subfrage der Studie ist, ob die Angstwerte der Studierenden im Fremdsprachenunterricht eine signifikante Veränderung in Abhängigkeit von der Variabilität des Deutschniveaus im Gymnasium aufweisen. Die One-Way-ANOVA Ergebnisse sind in Tabelle 9 dargestellt.

Tabelle 9: Vergleich der Angstwerte von Studierenden je nach Deutschniveau am Gymnasium

\begin{tabular}{|l|c|c|c|c|c|c|}
\hline Variablen & $\mathbf{n}$ & $\overline{\mathbf{X}}$ & $\mathbf{S}$ & $\mathbf{\text { sd }}$ & $\mathbf{F}$ & $\mathbf{p}$ \\
\hline ungenügend & 31 & 2.98 & 0.69 & $2-67$ & 2.73 & 0.72 \\
\hline ausreichend & 30 & 3.15 & 0.77 & & & \\
\hline gut & 9 & 3.65 & 0.89 & & & \\
\hline
\end{tabular}

Aus Tabelle 9 wird ersichtlich, dass sich die Angstwerte der Studierenden der Abteilung Deutsch als Fremdsprache auf Lehramt je nach dem Sprachniveau im Gymnasium nicht signifikant unterscheiden $[F(2-67)=2.73: p<0.05]$. Mit anderen Worten zeigt sich, dass das Niveau der Deutschkenntnisse am Gymnasium keinen Einfluss auf die Angstwerte der Studierenden im Fremdsprachenunterricht hat.

\subsection{Befunde des Interviewformulars}

Zur Unterstützung der quantitativen Daten wurde ein halbstrukturiertes Interviewformular erstellt, um von qualitativen Daten zu profitieren. Die Ergebnisse aus diesem Formular lauten wie folgt:

Im genannten Interviewformular wurde die Frage gestellt, ob die Studierenden der Abteilung Deutsch als Fremdsprache auf Lehramt Ängste haben oder nicht und was die Gründe dafür sein können. Diese Frage wurde in folgender Weise gestellt: „Haben Sie Angst in der Fremdsprache zu sprechen? Begründen Sie bitte." Einige Studierende, die mit „Ja“ geantwortet haben, stellen ihre Gründe wie folgt dar: 
S. 2 „Ich bin besorgt, weil ich nicht sicher bin, ob ich die Grammatik richtig verwende und ob die Wörter, die ich verwende, genau richtig sind."

S. 8„Es besteht die Angst, dass ich mich jemand anderem gegenüber nicht ausdrücken kann."

S. 9 "Ich bin besorgt, denn ich fühle mich nicht kompetent."

S. 11 „Ich bin in der Praxis nicht gut genug, weil ich im Alltag nicht spreche. Mein Wortschatz ist nicht ausreichend. Wir beschäftigen uns nicht genug mit der Sprache, die wir lernen."

S. 19 „Ich habe kein Selbstvertrauen."

S. 20 „Es passiert unwillkürlich. Denn der soziale Druck führt zu Schamgefühl. Dazu kommt noch das mangelnde Üben."

S. 21 "Ich vertraue meiner Aussprache nicht und habe eine soziale Angst. Wenn ich in der Fremdsprache antworte, bekomme ich die Antwort, Sie können die Sprache nicht, wir verstehen."'

S. 26 „Weil es im Türkischen keine Artikel gibt, habe ich immer das Gefühl, dass ich falsch spreche. Bevor ich spreche, überlege ich es mir mehrmals. Ich denke, dass meine Aussprache schlecht ist."

S. 30 „Ja, ich bin besorgt, dass ich die Sätze nicht richtig bilden oder ausdrücken kann."

S. 40 "Ich habe Angst, eine falsche Aussprache zu haben. Ich bin besorgt, dass jemand meine Sätze nicht versteht."

S. 45 „Ich denke, dass ich insbesondere in Deutsch, der Sprache und der Kultur immer noch fremd bin."

S. 52 "Ich bin besorgt, denn ich habe Angst, etwas falsch zu sagen. Dies hängt auch ein bisschen von der Klasse ab. Es gibt sehr viele in der Klasse, die Deutsch als Muttersprache sprechen und das macht mich nervös."

S. 53 „Ich bin besorgt, ich weiß, dass mein Niveau niedrig ist und dass ich erfolglos bin, weil ich nicht genügend übe."

S. 57 „Manchmal schäme ich mich, weil ich mich nicht richtig ausdrücken kann."

S. 59 „Ich bin besorgt, dass ich beim Sprechen die Grammatik nicht richtig verwenden kann und dass die Aussprache nicht richtig sein wird. Denn in der Klasse gibt es Muttersprachler und die machen mich nervös."

S. 61 „Dass ich die Wörter falsch ausspreche und mich nicht an die Wörter erinnere, erhöht mein Angstniveau."

S. 62 "Ich werde in der Klasse durch die Reaktionen der Muttersprachler unruhig. Ich bin besorgt wegen den Mitschülern die, anstatt zu helfen, Fehler suchen."

S. 65 „Es stört mich, nicht ganz richtig sprechen zu können. Ich denke, dass ich mangelndes Wissen und mangelnden Mut habe." 
Manche, die die Frage „Haben Sie Angst in der Fremdsprache zu sprechen? Begründen Sie bitte." mit „Nein“ beantwortet haben, haben ihre Gründe wie folgt ausgedrückt:

S. 1 "Andere machen auch Fehler in der Fremdsprache."

S. 5 „Ich bin nicht besorgt, nur nervt es mich, dass ich ständig das Gefühl habe, grammatische Fehler zu machen."

S. 7 „Ich bin nicht besorgt, denn ich denke nicht, dass die Menschen die Fremdsprache perfekt sprechen."

S. 10 "Ich habe keine Angst vor dem Lernen indem ich Fehler mache, im Gegenteil. Ich denke, dass ich so lerne."

S. 36 „Ich habe keine Angst in der Fremdsprache zu sprechen. Es macht mich nervös bei Menschen zu sprechen, die die Selbsteinschätzung vermitteln:,Ich spreche am besten."

S. 56 "Ich bin nicht besorgt, denn ich tue das Beste, um mich zu verbessern. Ich freue mich, wenn ich merke, dass ich im Sprechen besser werde."

S. 69 „Ich habe keine Angst Fehler zu machen. Aber wegen der faulen Typen in meiner Umgebung, wird über mein Sprechen meistens gelacht und deshalb habe ich keine Motivation. Ich bin meistens wegen der Angst im Sprechen der Anderen auch gehemmt."

Eine weitere Frage, die in der Studie gestellt wurde, lautet „Was sind die Gründe der Angst, die ihr Fremdsprachenlernen negativ beeinflussen?" Einige der Antworten lauten wie folgt:

S. 2 „Nicht selbstsicher sein und kein Selbstvertrauen haben."

S. 6 „Das Verhältnis zwischen Lehrer und Student, falsche Methode, mangelndes Üben und Wiederholen."

S. 8 „Unzureichender mündlicher Unterricht, in der Muttersprache durchgeführter Unterricht."

S. 13 "Die Angst, nicht zu bestehen und Fehler zu machen."

S. 19 „Plötzliche Fragen vom Lehrer."

S. 26 „Mein speaking Lehrer sagte mal: ,So einfach, und das kannst du nicht?' Das hat mich sehr getroffen."

S. 28 „Weil meine Grammatikkenntnisse sehr schlecht sind, fällt es mir schwer, die Grammatik einer anderen Sprache zu lernen." 
S. 32 „Für mich ist es ein negativer Faktor, dass der Lehrer von uns erwartet, wie ein Muttersprachler zu sprechen und somit auch das Niveau erhöht."

S. 35 "Mangelnder Wortschatz, grammatikalische Fehler."

S. 39 „Der Gedanke, dass ich nach meinem Studium arbeitslos werde, macht mich unwillig."

S. 40 "Mangelndes Selbstvertrauen. Ich vertraue mir nicht. Das Verhalten anderer, während ich spreche. Wenn sie lachen, während ich versuche einen Satz zu bilden."

S. 41 „Die Angst, vor der Klasse Fehler zu machen und sich zu blamieren."

S. 43 „Die Muttersprachler aus Deutschland machen es uns schwierig, im Unterricht zu lernen."

S. 46 "Stress, mangelndes Selbstvertrauen."

S. 48 "Vom Lehrer unter Druck stehen."

S. 51 "Aufregung, Umgebung, Vertrauen."

S. 53 „Wegen manchen Studenten, deren Sprachniveau hoch ist, kann ich meistens nicht fragen, was ich nicht verstanden habe."

S. 54 „Fremd gegenüber der Sprache sein."

S. 57 "Kein Beherrschen der alltäglichen Themen."

S. 60 "Gruppenzwang."

S. 61 "Aussprache und mangelnder Wortschatz."

S. 62 „Umgebung der Klasse, mangelndes Wissen."

S. 65 "Sich schämen, keinen Mut haben, kein Selbstvertrauen haben."

S. 66 „Die Klasse, der Freundeskreis, Angst haben Fehler zu machen, unzureichenden Wert darauf legen."

S. 68 „In der Türkei studieren."

S. 69 „Das Kritisieren von Freunden, obwohl sie selbst nicht einmal einen deutschen Satz bilden."

Eine andere Frage, um die quantitativen Daten in der Untersuchung zu unterstützen, lautet "Gibt es Maßnahmen in Ihren Fremdsprachenkursen, die Ihre Sprachangst beseitigen? Wenn ja, um welche handelt es sich?“ Die Antworten einiger Studierenden auf diese Frage lauten wie folgt:

S. 1 „Dieses Thema wird nicht so sehr behandelt."

S. 2 "Sie ermutigen uns mehr zum Sprechen."

S. 5 „Mehr oder weniger. Kleine Aktivitäten in der Klasse zum Deutschsprechen.”

S. 8 „Ich höre mir Musik in fremder Sprache an und bilde kurze Dialoge für mich.” 
S. 10 "Ich unterhalte mich immer in der Sprache, die ich lerne."

S. 12 "Ich versuche am Unterricht teilzunehmen und richtig oder falsch zu sprechen."

S. 13 „Bestimmte Kurse sind sprachorientiert und reduzieren somit die Angst."

S. 15 „Ich höre mir Menschen an, die Muttersprachler sind."

S. 20 "Es werden Übungen unternommen, um unsere Praxis zu verbessern."

S. 21 „Ich mache es persönlich. Ich sehe Filme, lese Bücher. Lerne Vokabeln, mache

Diktate, aber das dient nur dem Schriftlichen. Mein Hörverständnis hat sich verbessert, aber ich kann immer noch nicht sprechen."

S. 26 "Nein, es wird nichts gemacht."

S. 30 „Ja, es wird etwas gemacht. Motivationsgespräche.”

S. 32 „Man glaubt, dass dieses Problem durch viele Dialogübungen überwunden werden kann. Das glaube ich aber nicht. Unterschiedliche Übungen werden dazu nicht durchgeführt. Jeder bleibt auf seinem eigenen Niveau. Es gibt keinen Fortschritt."

S. 33 „Auch wenn die Schüler sprechen und Fehler machen, werden sie am Ende des Gesprächs korrigiert."

S. 40 „Es werden mehrere Aktivitäten durchgeführt. Beruhigende Gespräche werden geführt."

S. 45 „Der Unterricht ist nicht lehrer-, sondern schülerorientiert."

S. 51 „Anstatt fließend zu sprechen, buchstabieren sie oder helfen und beruhigen uns somit."

S. 54 „Es werden öfter sprachliche Übungen gemacht."

S. 57 „Sprechen und Präsentationen halten sind bestimmt nützlich aber ich denke nicht, dass es ausreichend ist."

S. 58 „Ich spreche mit einem Muttersprachler, dessen Sprache ich lerne und übe mit ihm."

S. 59 „Einige unserer Lehrer ermutigen uns."

S. 65 „In der Vorbereitungsklasse gab es Kommunikationskurse, in denen wir sehr oft sprechen konnten, deshalb war ich auch nicht besorgt. Aber im Lehramtsstudium sind diese Kurse weniger und die Blicke und Gedanken der Mitschüler machen mir Angst. Es werden keine Übungen dazu gemacht."

Die letzte Frage der Untersuchung lautet: „Was sind die Faktoren, die sich positiv auf Ihr Fremdsprachenlernen auswirken?" Im Folgenden einige der Antworten auf diese Frage: 
S. 1 „Etwas Neues lernen, Spaß haben wie mit einem Kreuzworträtsel, Sprachen lernen und sprechen gernhaben und weil es mir leichtfällt."

S. 2 "Wenn der Lehrer meine Antwort bestätigt."

S. 3 „Ausländische Serien und Filme, ausländische Lieder, ausländische Freunde."

S. 6 „Lehrer, die mir nahestehen und entsprechende Uhrzeiten der Kurse."

S. 7 „Der Wunsch, andere Kulturen kennen zu lernen.”

S. 8 "Ich liebe es, eine Fremdsprache zu lernen. Der Gedanke, mit Menschen anderer Kulturen in Kontakt zu kommen, gefällt mir."

S. 10 "Mein Erfolg im Unterricht prägt mein Üben."

S. 11 „Mein eigener Wille. Zu wissen, dass ich mich mit Leuten im Ausland verständigen kann und berufliche Möglichkeiten."

S. 14 „Der Gedanke, dass ich mit einer neuen Sprache eine neue Kultur kennenlerne, beeinflusst mich positiv."

S. 18 „Die gegenseitige Verständigung in der Fremdsprache steht an erster Stelle. Und das steigert meine Begeisterung für das Erlernen einer Fremdsprache."

S. 21 „Internet, Technologie, verschiedene Bücher."

S. 26 „Ich versuche mich zu motivieren, indem ich daran denke, dass ich Lehrer werde."

S. 29 "Ich mag es nicht, eine Fremdsprache zu lernen. Ich fühle mich nicht wie ein neuer Mensch, wenn ich in einer anderen Sprache spreche."

S. 31 „Der erste Faktor, der mich motiviert ist meine eigene Motivation und mein Perfektionismus. Und die motivierenden Sätze meiner Lehrer."

S. 32 „Unterhaltsame Aktivitäten und Übungen im Fremdsprachenunterricht, die unser Lernen prägen. Ein Lehrer ohne hohe Erwartungen, ein ruhiges Arbeitsfeld und die Klassenordnung."

S. 38 "Der Wunsch ins Ausland zu gehen."

S. 39 „Es gefällt mir, eine neue Sprache, Kultur und ein neues Land kennen zu lernen. Deshalb begeistert mich das Sprachenlernen."

S. 40 „Musik in der Fremdsprache hören, Videos oder Filme sehen. In der Fremdsprache Berichte, Geschichten lesen, Nachrichten sehen."

S. 48 „Die Haltung des Lehrers, unterhaltsame Kurse."

S. 52 „Ich habe Träume, wie zum Beispiel in der Zukunft ins Ausland zu gehen, das motiviert mich. Und außerdem möchte ich sehr gerne Freunde aus anderen Ländern haben."

S. 53 „Die Tatsache, dass die Lehrer nicht schimpfen, uns gegenüber verständnisvoll sind und uns nicht immer mit einem hohen Niveau vergleichen."

S. 57 „Motivation, Lust zum Lernen bekommen, verschiedene Kulturen und natürlich eine andere Art von Kommunikation." 
S. 60 „Die Motivation des Lehrers, die Sprache auf das Niveau des Lernenden zu übertragen, indem die Sprache vereinfacht wird."

S. 62 "Ermutigung des Lehrers, Hilfe, moderate Herangehensweise."

S. 63 „Der Wille etwas Neues zu lernen, die Kultur und Struktur des Landes oder Länder kennenzulernen, deren Sprache man lernt."

S. 67 "Dass es weltweit in allen Arbeitsbereichen nützlich sein wird."

S. 68 „Mit Menschen anderer Sprachen zu kommunizieren."

Die Studierenden der Abteilung Deutsch als Fremdsprache auf Lehramt haben die obigen Faktoren genannt, die sich durch den Abbau von Ängsten positiv auf das Erlernen von Fremdsprachen auswirken. Schlussfolgerungen und Vorschläge zu diesen Ergebnissen werden im nächsten Abschnitt vorgestellt.

\section{Schlussfolgerung}

Ziel dieser Studie war es, die Angstzustände im Fremdsprachenunterricht der Studierenden der Abteilung Deutsch als Fremdsprache auf Lehramt der Universität Anadolu zu ermitteln. Diese Studie soll zugleich untersuchen und dabei herausfinden, ob sich diese Angstzustände in Bezug auf verschiedene Variablen signifikant unterscheiden. Die Ergebnisse der Studie zeigen, dass das allgemeine Niveau der Angstzustände im Fremdsprachenunterricht bei den Studierenden moderat ist. Dies kann daran liegen, dass die Studierenden zwar Fremdsprachenunterricht erhalten, sich aber nicht als kompetent genug betrachten. Der am häufigsten auftretende gemeinsame Ausdruck der Studierenden, „Es stört mich jedoch nicht, dass die Anzahl der Deutschstunden erhöht werden", lässt davon ausgehen, dass die Studierenden eine positive Einstellung zum Deutschunterricht haben. In der Studie wurde getestet, ob sich die Angstzustände der Studierenden im Fremdsprachenunterricht in Bezug auf verschiedene Variablen unterscheiden. Es wurde der Entschluss gezogen, dass einige Variablen einen Einfluss auf die Angst hatten und andere die Angst nicht beeinflussten. Die erste Variable, die keinen Einfluss auf die Angst vor dem Fremdsprachenunterricht hat, ist das Geschlecht. Aus den Ergebnissen der Studie kann geschlossen werden, dass sich die Ängste vor dem Fremdsprachenunterricht in den Geschlechtsvariablen nicht unterscheiden. Basierend auf diesem Befund kann der Entschluss gezogen werden, dass die Angstzustände im Fremdsprachenunterricht bei weiblichen und männlichen Studierenden ähnlich sind. Eine andere Variable, die keinen Einfluss auf die Angst hat, ist die Art des besuchten Gymnasiums. Es wurde kein signifikanter Unterschied zwischen den Angstwerten der 
Studierenden festgestellt, die einen Abschluss von unterschiedlichen Arten von Gymnasien hatten. Unter Arten von Gymnasien sollen hier naturwissenschaftliches Gymnasium, Anadolu Gymnasium, Fachgymnasium u.a. verstanden werden. Durch die Studie gewinnt man die Erkenntnis, dass die Angstwerte der Studierenden im Fremdsprachenunterricht nicht von den Variablen des Schultyps beeinflusst werden. Eine weitere Variable, die sich nicht auf die Angst auswirkte, war das Sprachniveau der Studierenden an den Gymnasien. Es wurde festgestellt, dass es keinen Unterschied zwischen den Angstwerten der Studierenden im Fremdsprachenunterricht gab, die ihr Sprachniveau am Gymnasium als ungenügend, ausreichend oder gut definierten. Basierend auf diesem Befund wurde der Entschluss gezogen, dass das zuvor erworbene Fremdsprachenniveau keinen Einfluss auf die Angst vor dem Fremdsprachenunterricht hat.

In der Studie wurden auch Variablen ermittelt, die die Angstwerte in Bezug zum Fremdsprachenunterricht beeinflussen. Den Befunden zufolge gibt es einen signifikanten Unterschied zwischen den Fremdsprachen-Angstwerten Studierenden, die im Ausland gelebt haben und Studierenden, die nicht im Ausland gelebt haben. Nach diesen Erkenntnissen waren die Angstwerte von Studierenden, die im Ausland waren höher als die Angstwerte von Studierenden, die nicht im Ausland waren. Auf der Grundlage dieser Erkenntnis kann gesagt werden, dass die Bedenken hinsichtlich des Fremdsprachenunterrichts höher sind als die Erwartung von Studierenden, die im Ausland korrekt sprechen, und der Gedanke, dass sie sich schämen, weil sie Gleichaltrigen und Dozenten gegenüber Fehler machen. Eine weitere bedeutende Erkenntnis, die sich aus der Studie ergibt, besteht darin, dass es einen signifikanten Unterschied zwischen den Fremdsprachenangstwerten von Studierenden, die eine Schule im Ausland besucht haben, und Studierenden, die keine Schule im Ausland besucht haben, gibt. Aufgrund der Forschungsergebnisse zeigt sich, dass die Angst vor dem Fremdsprachenunterricht bei Studierenden, die eine Schule im Ausland besucht haben, größer ist als bei Studierenden, die keine Schule im Ausland besucht haben. Basierend auf der Idee, dass Studierende, die aus ähnlichen Gründen im Ausland studieren, die Zielsprache genauer und flüssiger verwenden sollten, kann gesagt werden, dass sich diese beiden Variablen gegenseitig unterstützen. Eine weitere Variable, die in der Studie zur Untersuchung kam und sich auf die Angst vor dem Fremdsprachenunterricht auswirkt, ist die Variable über den Erhalt einer vorbereitenden Ausbildung an der Universität. Wenn die Ergebnisse der Studie untersucht werden, zeigt sich, dass die Fremdsprachenangstwerte der Studierenden, die keine vorbereitende Ausbildung an der Universität absolvieren, höher 
sind als die der Studierenden, die eine vorbereitende Ausbildung an der Universität absolvieren. Mit anderen Worten waren die Angstzustände im Fremdsprachenunterricht bei Studierenden, die keine vorbereitende Ausbildung erhalten hatten, höher als bei Studierenden, die eine vorbereitende Ausbildung erhalten hatten. Auf der Grundlage dieses Ergebnisses kann der Entschluss gezogen werden, dass in den Abteilungen, die Fremdsprachenunterricht anbieten, eine vorbereitende Ausbildung sehr wichtig ist.

Aus den Ergebnissen der qualitativen Fragen zur Untermauerung der quantitativen Daten geht hervor, dass die Angstursachen der Studierenden sowohl auf interne als auch auf externe Gründe zurückzuführen sind. Aus den Forschungsergebnissen geht hervor, dass es interne Gründe wie mangelndes Selbstvertrauen und Scham gibt, durch die sich die Studierenden schlecht fühlen, z.B. falsche Grammatikregeln, falsche Aussprache, falsche Wortauswahl und Angst. Darüber hinaus kann gesagt werden, dass externe Faktoren wie die Angst, von Freunden verspottet und von Gleichaltrigen negativ kritisiert zu werden, Gründe für die Besorgnis der Studierenden über die Fremdsprache darstellen. Bei der Frage nach den Ursachen von Ängsten, die sich negativ auf das Lernen auswirken, antworteten die Studierenden in ähnlicher Weise: mangelndes Selbstvertrauen, Angst vor Erfolglosigkeit, Mangel an Vokabeln, Angst vor grammatikalischen Fehlern und ebenfalls daran zu glauben, dass sie sich gegenüber Gleichaltrigen blamieren würden. Fischer (2005, S. 43) kam zu einem ähnlichen Ergebnis in ihrer Arbeit und drückte dies wie folgt aus: „Charaktereigenschaften wie Extrovertiertheit bzw. Introvertiertheit können nicht automatisch mit dem Erfolg in der mündlichen Produktion einer Fremdsprache gleichgesetzt werden, dennoch ist auffallend, dass Studierende selbst angeben, dass sie aufgrund ihrer Schüchternheit Schwierigkeiten in dem Bereich haben".

Auf die Frage, ob es Maßnahmen zur Verringerung der Angst in Fremdsprachenunterricht gibt, gaben einige der Studierenden an, dass es keine Maßnahmen gibt, einige gaben jedoch an, dass die positiven Einstellungen und Anstrengungen der Lehrkräfte wie etwa das Sprechen, das Lesen von Büchern, das Ansehen von Filmen und das Hören von Musik die Angst reduzieren. Der letzte Befund der Studie bezieht sich auf die Faktoren, die sich positiv auf das Erlernen einer Fremdsprache auswirken. Diese Faktoren sind, eine neue Sprache und Kultur lernen, Filme, Lieder usw. in einer Fremdsprache verstehen können, ins Ausland gehen, Berufschancen, mit Menschen anderer Herkunft und Sprache kommunizieren. Ebenfalls dazu zählen die positiven Einstellungen und Rückmeldungen von Fakultätsmitgliedern, die das Erlernen einer Sprache positiv beeinflussen. Nach den erhaltenen Befunden der 
Untersuchung ist festzustellen, dass die Studierenden vor allem beim Sprechen große Ängste haben und die Gründe dafür sowohl interne als auch externe Faktoren sein können. Die Angst der Lernenden muss reduziert werden, denn Angst wirkt sich direkt auf den Lernprozess aus und gilt im Bereich des Fremdsprachenunterrichts grundsätzlich als besondere Reaktion (Süleymanova, 2011, S. 56).

Nach einer allgemeinen Bewertung der Forschungsergebnisse wird deutlich, dass die pädagogischen Fakultäten es benötigen umgestaltet und verbessert zu werden, damit die Angstzustände von Studierenden der Abteilung Deutsch als Fremdsprache auf Lehramt, die sich auf einem mittleren Niveau befinden, auf ein Minimum reduziert werden. Um dies zu erreichen, sollten zunächst die Ursachen dieser Bedenken detaillierter untersucht werden. Um die Ursachen feststellen zu können, die durch den Gruppenzwang der Kommilitonen oder die Angst davor, Fehler zu machen, entstehen, sollten Untersuchungen im Unterricht durchgeführt werden. Da die vorliegende Untersuchung ausschließlich mit Studierenden des 2. Studienjahres und mit nur 6 Variablen durchgeführt wurde, ist es außerdem wichtig, einen Beitrag zur Literatur zu leisten, indem künftige Untersuchungen mit allen Studierenden der Abteilung Deutsch als Fremdsprache auf Lehramt und mit verschiedenen Variablen durchgeführt werden. Eine gründliche Untersuchung dieser und vieler anderer Faktoren, die sich negativ auf das Lernen auswirken, ist für effektivere Fremdsprachenunterrichte von größter Bedeutung, da die Absolventen der Pädagogischen Fakultät die zukünftigen Lehrkräfte werden und selbstbewusste Lehrkräfte zweifellos noch erfolgreichere Generationen erziehen.

\section{Begutachtung: Extern begutachtet.}

Interessenkonflikt: Es besteht kein Interessenkonflikt.

Finanzielle Förderung: Dieser Beitrag wurde von keiner Institution finanziell unterstützt.

Peer-review: Externally peer-reviewed.

Conflict of Interest: The authors have no conflict of interest to declare.

Grant Support: The authors declared that this study has received no financial support.

\section{Literaturverzeichnis}

Aida, Y. (1994). Examination of Horwitz, Horwitz, and Cope's construct of foreign language anxiety: The case of students of Japanese. The modern language journal, 78(2), 155-168.

Aydın, S. \& Zengin, B. (2008). Yabancı dil öğreniminde kaygı: Bir literatür özeti. Journal of Language and Linguistic Studies, 4(1), 81-94. 
Azher, M., Anwar, M. N. \& Naz, A. (2010). An investigation of foreign language classroom anxiety and its relationship with students' achievement. Journal of College Teaching and Learning, 7(11), 33-40.

Balemir, S. H. (2009). The sources of foreign language speaking anxiety and the relationship between proficiency level and degree of foreign language speaking anxiety. (Doctoral Dissertation). Bilkent University, Turkey.

Batumlu, D. Z. \& Erden, M. (2007). Yıldız Teknik Üniversitesi Yabancı Diller Yüksek Okulu hazırlık öğrencilerinin yabancı dil kaygıları ile İngilizce başarıları arasındaki ilişki. Eğitimde kuram ve uygulama, 3(1), 24-38.

Bekleyen, N. (2004). Foreign language anxiety. Çukurova Üniversitesi Sosyal Bilimler Enstitüsü Dergisi, 13(2), 27-39.

Can, E. \& Can, C. I. (2014). Türkiye'de ikinci yabancı dil öğretiminde karşılaşılan sorunlar. Trakya Üniversitesi Eğitim Fakültesi Dergisi, 4(2), 43-63.

Doğan, Y. \& Tuncer, M. (2016). Examination of foreign language classroom anxiety and achievement in foreign language in Turkish University students in terms of various variables. Journal of Education and Training Studies, 4(5), 18-29.

Dörnyei, Z. (1994). Motivation and motivating in the foreign language classroom. The Modern Language Journal, 78(3), 273-284.

Duman, B., Göral, G. N. \& Bilgin, H. (2017). Üniversite öğrencilerinin sınıf ortamında yabancı dil konuşma kaygısı üzerine nitel bir çalışma. Adnan Menderes Üniversitesi Eğitim Fakültesi Eğitim Bilimleri Dergisi, 8(2), 13-27.

Fischer, S. (2005). Sprechmotivation und Sprechangst im DaF-Unterricht. German as a foreign language. 3, 31-45.

Gedik, M. (2015). Siirt örnekleminde ortaöğretim öğrencilerinin konuşma kaygılarının incelenmesi. Sosyal Bilimler Araştırmaları Dergisi, 5(13), 77-93.

Genç, G. (2009). İnönü Üniversitesi Yabancı Diller Yüksekokulu öğrencilerinin yabancı dil kaygıları. e-Journal of New World Sciences Academy, 4(3), 1080-1089.

Horwitz, E. K., Horwitz, M. B. \& Cope, J. (1986). Foreign language classroom anxiety. The Modern language journal, 70(2), 125-132.

İşigüzel, B. \& Kırmızı, B. (2014). Lise öğrencilerinin Almanca öğrenme sürecinde başarısızlık nedenlerinin değerlendirilmesi. Kafkas Üniversitesi Sosyal Bilimler Enstitü Dergisi, 1(13), 45-63.

Kim, S. Y. (2009). Questioning the stability of foreign language classroom anxiety and motivation across different classroom contexts. Foreign Language Annals, 42(1), 138-157.

Matsuda, S. \& Gobel, P. (2004). Anxiety and predictors of performance in the foreign language classroom. System, 32(1), 21-36.

Oflaz, A. (2019). Almanca hazırlık sınıfı öğrencilerinin kaygı, dil öğrenme stratejileri kullanımları ile akademik başarıları arasındaki ilişki. Diyalog interkulturelle Zeitschrift für Germanistik, 7(2), 305-319.

Önem, E. (2010). The relationship among state-trait anxiety, foreign language anxiety and test anxiety in an EFL setting. Dil Dergisi, 148, 17-36.

Öner, G. \& Gedikoğlu, T. (2007). Ortaöğretim öğrencilerinin İngilizce öğrenimlerini etkileyen yabancı dil kaygısı. Gaziantep Üniversitesi Sosyal Bilimler Dergisi, 6(2), 144-155. 
Angstzustände im Fremdsprachenunterricht von Studierenden der Abteilung Deutsch als Fremdsprache auf Lehramt ...

Öztürk, G. \& Gürbüz, N. (2014). Speaking anxiety among Turkish EFL learners: The case at a state university. Journal of Language and Linguistic Studies, 10(1), 1-17.

Rodríguez, M. X. \& Abreu, O. (2003). The stability of general foreign language classroom anxiety across English and French. The Modern Language Journal, 87(3), 365-374.

Sparks, R. L. \& Ganschow, L. (2007). Is the foreign language classroom anxiety scale measuring anxiety or language skills? Foreign Language Annals, 40(2), 260-287.

Süleymanova, R. (2011). Abbau der Sprechangst im Unterricht Deutsch als Femdsprache: Eine empirische Untersuchung am Beispiel der Integrationskurse. Berlin: Köster.

Şener, S. (2015). Yabancı dil öğrenme kaygısı ve başarı: Çanakkale Onsekiz Mart Üniversitesi öğrencilerinin durum değerlendirmesi. Turkish Studies-International Periodical for the Languages, Literature and History of Turkish or Turkic, 10(3), 875-890.

Trylong, V. L. (1987). Aptitude, attitudes, and anxiety: A study of their relationships to achievement in the foreign language classroom. (Doctoral Dissertation). Purdue University, USA.

Tüm, D. Ö. \& Kunt, N. (2013). Speaking anxiety among EFL student teachers. Hacettepe Üniversitesi Eğitim Fakültesi Dergisi, 28(3), 385-399.

Yılmaz, D. \& Maden, S. S. (2016). Dil öğrenim sürecinde Almanca öğretmen adaylarının kaygı tutumlarına ilişkin bir araştırma. Trakya Üniversitesi Eğitim Fakültesi Dergisi, 6(2), 201-211.

Young, D. J. (1991). Creating a low-anxiety classroom environment: what does language anxiety research suggest? The modern language journal, 75(4), 426-437.

Zerey, Ö. G. (2008). Tiyatro uygulamasının öğrencilerin yabancı dil konuşma kaygısı üzerindeki etkisi. (Yüksek Lisans Tezi). Mustafa Kemal Üniversitesi, Türkiye.

Zhanibek, A. (2001). The relationship between language anxiety and students' participation in foreign language classes. (Doctoral Dissertation). Bilkent University, Turkey. 